\title{
Surgical Management of Portal Biliopathy - A Prospective Analysis.
}

\author{
S.P Girish ${ }^{\circledR 1}$, Jagan Mohan B Reddy ${ }^{\odot 2}$ \\ ${ }^{1}$ Associate Professor, Department of Surgical Gastroenterology, Sapthagiri Institute of Medical Sciences and Research Centre, Bangalore, Karnataka, India, ${ }^{2}$ Assistant \\ Professor, Department of Surgical Gastroenterology, Sapthagiri Institute of Medical Sciences and Research Centre, Bangalore, Karnataka, India.
}

\section{Abstract}

Background: Portal biliopathy denotes intrahepatic and extra hepatic biliary ductal abnormalities in portal hypertension. It is usually associated with extra hepatic portal vein obstruction (EHPVO). These patients are also prone to develop obstructive jaundice as a result of strictures and/or choledocholithiasis. Surgical management of obstructive jaundice in such patients becomes difficult in the presence of these collaterals. The aim of the study is to prospectively analyze the approach to management of patients with Symptomatic portal biliopathy. Subjects and Methods: The study was conducted at Narayana Medical College \& Hospital, Chintareddy Palem, Nellore, Andhra Pradesh on surgical management of the patients of EHPVO with portal biliopathy presenting to the surgical clinic of this tertiary referral center between November 2016 and October 2017. The data was analyzed for presentation, clinical features, imaging and results of surgical management. Results: During the study period, total of 44 patients of EHPVO were referred for surgical management. Of these 14 patients ( 9 males, mean age 34.6 years) were diagnosed to have portal biliopathy. Ten patients had prior history of variceal bleed which was managed endoscopically. Jaundice was the most common symptom followed by right upper quadrant pain and recurrent cholangitis. Four patients had prior unsuccessful endoscopic management. ERCP / MRCP was used for delineation of the biliary tree, which showed irregularity (14 patients), dominant strictures (8 patients), filling defects (5 patients), and intrahepatic biliary dilatation (7 patients). Proximal splenorenal shunt (PSRS) was performed in 13 patients. While in 1 patient peroperatively liver was found to be grossly nodular, hence gastro-esophageal devascularization with simultaneous biliary drainage was done. Of the 13 patients who underwent PSRS, all patients were intensively followed for 4-6 weeks with history and liver function tests. After 6 weeks, five patients showed clinical as well as biochemical improvement and they are being followed up regularly. Eight patients had persistent symptoms and abnormal liver function tests. These were the patients with dominant stricture and choledocholithiasis. Of these, 6 patients underwent Roux-en-Y hepaticojejunostomy. The average blood transfusion requirement at second surgery was 1 unit. Postoperative complications were minimal with no mortality. One patient and was lost to follow up and the remaining one is awaiting second surgery (RYHJ). Over a follow up of 3-28 months the patients are asymptomatic and well. Conclusion: Portal biliopathy with symptomatic biliary obstruction needs intervention. Surgical decompressive shunt followed by biliary drainage is the best possible treatment. While for most of the early biliary changes shunt alone is effective, patients with dominant stricture will need a biliary diversion which can be safely performed following Porto systemic shunt without increase in morbidity or mortality.

Keywords: Portal biliopathy, Intrahepatic duct, Extrahepatic duct

Corresponding Author: Jagan Mohan B Reddy, Assistant Professor, Department of Surgical Gastroenterology, Sapthagiri Institute of Medical Sciences and Research Centre, Bangalore, Karnataka, India.

E-mail: sweetjagan20204u@gmail.com

Received: 25 April 2020

Revised: 27 May 2020

Accepted: 16 June 2020

Published: 5 July 2020

\section{Introduction}

Portal biliopathy, the terminology used to describe biliary ductal and gallbladder wall abnormalities seen in patients with portal hypertension. ${ }^{[1]}$ The changes of portal biliopathy have been reported to be more common in patients with portal vein thrombosis than in patients with non-cirrhotic portal fibrosis or cirrhosis of the liver. Unless the clinical and radiological profile of this entity is well appreciated, the condition could be overlooked. The collaterals around extra hepatic bile duct, by virtue of their proximity cause compression leading to Portal biliopathy. These morphological changes on cholangiogram viz. caliber irregularities, indentations, strictures, dilatation, ectasia, intraluminal filling defects and nodularity, have been termed as portal biliopathy, pseudosclerosing cholangitis or Peudocholangiocarcinoma. Apart from extrinsic compression, ischemia has also been proposed to contribute to the development of biliary strictures. Although these changes are seen in $80-100 \%$ of the patients with EHPVO only $5-14 \%$ are symptomatic. ${ }^{[1-5]}$ These changes become significant to give rise to overt obstructive jaundice and possibly contribute to 
the development of choledocholithiasis. ${ }^{[4]}$ The patients with symptomatic portal biliopathy have jaundice, cholangitis or right upper quadrant pain. These symptomatic patients need prompt relief of biliary obstruction otherwise there is increased morbidity and mortality related to cholangitis and secondary biliary cirrhosis.

The first description of symptomatic biliary obstruction in portal hypertension was reported in 1944 by Fraser et. Then onwards there are few case reports and case series on defining the disease and management. It was not recognized until the proper description as a separate complication of portal hypertension by Sarin et al and Dilawari et al in 1992. ${ }^{[1,2]}$ Since then there are several attempts on defining the symptomatology and treatment options. In 1998, Choudhary. A et al suggested the treatment criteria for portal biliopathy. In this study we analyse the institutional experience of prospectively collected data with reference to surgical management and outcome.

\section{Subjects and Methods}

The study was conducted at Narayana Medical College \& Hospital, Chintareddy Palem, Nellore, Andhra Pradesh on surgical management of the patients of EHPVO with portal biliopathy presenting to the surgical clinic of this tertiary referral center between November 2016 and October 2017. The demographic detail, clinical presentation, relevant past medical history, investigations, nature of biliary obstruction, management and outcome in these patients was analyzed. As a part of protocol, the study period was divided into two phases. In the initial phase, the demographic details, clinical presentation and relevant past medical history were recorded. All patients underwent investigations to confirm the diagnosis of EHPVO and portal biliopathy. Ultra sound abdomen combined with Doppler was used to assess the portal venous system and either ERCP (done elsewhere) or MRCP for visualization of biliary anatomy. All these patients underwent proximal splenorenal shunt after preoperative preparation except for one patient who had gross nodular liver preoperatively. Post-operative morbidity and mortality were documented. These patients were intensively followed up for 6 weeks. During follow up patients complaints were recorded and physical examination and LFT were performed. At the end of 6 weeks all investigations were repeated including UGIE, Doppler and MRCP. The changes were noted and recent MRCP was compared with the prior imaging. In the second phase of the study, those patients who were still having dominant biliary strictures underwent hepaticojejunostomy after variable period depending on follow up, to the hospital after 6 weeks. During this phase intraoperative details, duration of surgery and blood loss and blood transfusion requirements were specially recorded. After second surgery again patients were followed up till date for further developments.
All patients were documented cases of EHPVO with portal cavernoma demonstrated on ultrasonography (US). Portal vein thrombosis and presence of collaterals were confirmed by color Doppler in all 14 patients. One patient with chronic pancreatitis underwent a computed tomography (CT) of the abdomen, which confirmed the diagnosis of EHPVO. Malignancy was excluded by findings on imaging and followup. Cirrhosis was excluded on the basis of absence of risk factors, history of significant alcohol intake, markers for hepatitis $\mathrm{B}$ and $\mathrm{C}$ viruses and clinical course on follow-up. Apart from one patient with chronic calcific pancreatitis, no patient had any obvious risk factors for portal vein thrombosis. Results of hematological investigations for pro-thrombotic disorders were, however, not available for all patients. No patient was on anticoagulant therapy. The biliary imaging ERCP or MRCP were reviewed. Postoperative morbidity, mortality and long-term outcome on follow up were analyzed.

\section{Results}

\section{Clinical presentation and investigations}

There were nine males and five females (median age 35 years; range 14-56 years). All patients had jaundice which was progressive in eight and fluctuating in six patients. Duration of jaundice ranged from 6 to 180 months (median 2 years). History of cholangitis (diagnosed by the presence of fever along with jaundice and relieved after antibiotic treatment with or without biliary drainage) was present in eight patients. The number of episodes of cholangitis varied from one to seven (median two). Five patients had prior ERC and stenting for acute cholangitis not responding to antibiotics; they required repeat stenting of average 1 per year. One had symptomatic hypersplenism (recurrent epistaxis, platelet count $-50000 /$ cumm or white blood cell count [WBC] count $-2500 /$ cumm).

Ten patients had a past history of upper gastrointestinal (UGI) bleed. All these patients were on an Endoscopic variceal eradication program and adequately controlled. The bleeding preceded jaundice by a variable period of 1-10 years (mean 7.4 years). Jaundice was the sole manifestation of EHPVO in 4 patients. All these four patients had recurrent episodes of jaundice for duration of 6 months -15 years before the diagnosis of EHPVO. In all these patients, EHPVO was diagnosed during evaluation for jaundice. All these patients had esophageal varices on endoscopy but had not received any prophylactic sclerotherapy. Two patients were on beta blocker prophylaxis One patient with chronic pancreatitis (CP), portal vein thrombosis and portal cavernoma, had recurrent episodes of jaundice for 11 years and she was asymptomatic for chronic pancreatitis.

The clinical parameters of these patients are shown in [Table $1]$. 


\begin{tabular}{ll}
\hline \multicolumn{2}{l}{ Table 1: Clinical Parameters } \\
\hline Parameters & Numbers (percentage) \\
\hline Age (mean) & 35 yrs (14-56) \\
Sex (M:F) & $9: 5$ \\
Jaundice & $14(100 \%)$ \\
Recurrent Cholangitis & $8(57.6 \%)$ \\
Recurrent pain RUQ* & $10(71.4 \%)$ \\
H/o GI bleed & $10(71.4 \%)$ \\
$\begin{array}{l}\text { Symptomatic hyper- } \\
\text { splenism }\end{array}$ & $1(7.1 \%)$ \\
\hline
\end{tabular}

*RUQ: right upper quadrant

Past history of umbilical sepsis was present in three patients. Two other patients had history of abdominal surgery 10 year and 12year back respectively. None of these patients had history typical of acute portal vein thrombosis. There were no other known etiological factors for cause of EHPVO found in remaining patients.

Clinical examination revealed Jaundice in 10 patients (71\%) and splenomegaly in all patients. Apart from this hepatomegaly was found in 8 patients, which may be attributed to congestive liver due to biliary obstruction. One patient had mild ascites but did not have any other signs of cirrhosis. Other findings included umbilical hernia in 2, abdominal scars in 2 and pedal oedema.

\section{Investigations}

Liver function tests (LFT) was documented in all patients at admission. Serum Bilirubin and alkaline phosphatase (ALP) was elevated in most patients. Mean serum Bilirubin was $4.06 \mathrm{mg} / \mathrm{dl}$ (range $0.5-18.3 \mathrm{mg} / \mathrm{dl}$ ). Few patients had normal LFTs but had documented elevated Bilirubin during attack of jaundice. Mean ALP value was 304.46 IU (range 80-571 IU).Most of the patients had normal liver AST and ALT values. Serum Albumin was in the range of $3 \mathrm{gm} / \mathrm{dl}-4.2 \mathrm{gm} / \mathrm{dl}$ suggesting normal liver function in these patients.

All these patients underwent other hematological and biochemical parameters. Prothrombin time was recorded and corrected if it was abnormal. The radiological investigations included Ultrasound and Doppler abdomen, and Magnetic resonance cholangiogram. Few patients had ERC done else where hence the same was used for decision making. The USG and MRC/ERC findings are given in the following tables USG finding in the patients studied

\section{Biliary imaging}

Even though MRCP was the preferred modality of choice in our study, few patients already had ERCP and stenting done in the resent past. Hence these ERCP images were used in characterization of biliary abnormality. Those patients

\begin{tabular}{|ll|}
\hline \multicolumn{1}{|c|}{ Table 2: Investigations } \\
\hline Findings on USG & Number (\%) \\
\hline Gall stones & $5(35 \%)$ \\
CBD stones & $8(57 \%)$ \\
\hline IHBRD & 12 \\
Cbd dilatation & 13 \\
Liver echoe alteration & 3 \\
\hline
\end{tabular}

CBD-common bile duct, IHBRD-intrahepatic biliary radical dilatation.

Table 3: $\mathrm{MRC} / \mathrm{ERC}$ findings of the patientsCholangeographic findings in 14 patients

\begin{tabular}{|c|c|}
\hline Common bile duct changes & Numbers \\
\hline $\begin{array}{l}\text { Irregularity of outline Caliber irregular- } \\
\text { ity Indentation Angulation }\end{array}$ & 7832 \\
\hline $\begin{array}{l}\text { Focal narrowing/stricture Number Site } \\
\text { Ectasia/dilatation Filling defects }\end{array}$ & $\begin{array}{l}8 \text { BBS type1- } \\
\text { 1,type II-5,type } \\
\text { III-1,type IV-1 } \\
68\end{array}$ \\
\hline $\begin{array}{l}\text { Common hepatic duct changes } \\
\text { Irregularity of outline Focal narrow- } \\
\text { ing/stricture Dilatation }\end{array}$ & 3110 \\
\hline $\begin{array}{l}\text { Intra-hepatic biliary radicals Dilatation } \\
\text { Crowding }\end{array}$ & 121 \\
\hline
\end{tabular}

first seen in our department with suspected portal biliopathy underwent MRCP examination. Both imaging modalities were equally effective in delineating the anatomy. ERC/MRC findings are given in Table 10. Cholangeography showed common bile duct strictures in eight patients (BBS type11, type II-5, type III-1,type IV-1) and CBD stones in eight patients.

Doppler and upper GI endoscopy was performed to confirm the portal hypertension and the cause i.e. EHPVO. Doppler examination was helpful in assessing the splenic vein size which is important for shunt surgery. All patients had portal cavernoma. Mean splenic vein size was $8.7 \mathrm{~mm}$. UGI endoscopy revealed esophageal varices in 10 patients, obliterated in 4 patients. The grade of varices range was 13.Few patients also had Gastro esophageal and Ectopic varices within stomach.

None of the patients on clinical and investigative parameters were found to have cirrhosis.

\section{Management}

All patients underwent surgical intervention. As mentioned earlier the management was divided into two phases. In the initial phase proximal splenorenal shunt (PSRS) was attempted in all patients. PSRS is the preferred shunt procedure at our institution for management of EHPVO. As a total shunt it 
decompresses the whole portal system hence it is the logical option in patients with portal biliopathy. As per procedure, after preoperative preparation all patients were taken up for surgery. Left sub costal incion was used for access. In the initial evaluation, liver texture, ascites splenic size, collateral distribution were noted. Splenectomy was done and end to side PSRS was performed in 13 patients. One patient had shrunken nodular liver and under went devascularization and side to side hepatico jejunostomy. True cut Liver biopsy was taken from both the lobes in all patients. Total blood loss and blood transfusion requirement were noted which was 0.5 unit average.

Post operatively one patient required exploration for intra abdominal bleeding on day one. 3 patients had superficial wound infection and two patients had transient ascites which responded to diuretics. There were no post operative mortalities. Average hospital stay was 4.4 days (range 3-9 days). Liver biopsy showed marked fibrosis in three patients.

All patients were followed up at two weekly intervals for minimum of 6 weeks. During follow up complaints were noted. LFT was performed. The LFT pattern in patients with stricture was compared with those without strictures at the time of first operation. The following graph shows persisting altered LFT in the range of $3.4-5.7 \mathrm{mg} / \mathrm{dl}$ in the second group with persisting strictures compared to near normal results in second group.

During follow up 3 patients had single episode of cholangitis and were managed with antibiotics. One patient developed liver failure and died after 4 months of shunt surgery. This patient was not on regular follow up as he was from far of place. His liver biopsy had revealed secondary biliary cirrhosis. At six weeks all patients underwent Doppler and UGI endoscopy for shunt patency and diminution of esophageal varices. variceal size was diminished at least by 1 grade uniformly an all patients. Regarding shunt patency, Doppler did not visualize the shunt and blood flow through the shunt in majority of the patients due to overlying bowel gas shadow. However the hapato fugal blood flow and decreased collaterals compared to previous examination was used as an indirect evidence of shunt patency. In our study Doppler showed shunt patency in only two patients, however the indirect evidence described above was present in all 11 remaining patients. Those patients without any dominant biliary abnormalities and symptoms were followed up at 3 month intervals for a period of 7 to 17 months. None of these patients had variceal bleeding or any biliary symptoms and required no further interventions.

Those patients with dominant strictures and those with persisting symptoms underwent ultrasound examination followed by MRCP at a variable period from 6weeks to 6 months depending on their follow up. There were total eight patients in this category. However one patient refused further treatment and the other patient is awaiting MRCP examination. In these 6 patients MRCP revealed the structural abnormalities similar to the previous examination (before shunt surgery). Only one patient showed progression of stricture from BBS2 to BBS 3.None showed any marked improvement in the biliary abnormality. Two patients without any dominant structural abnormality underwent MRCP and showed remarkable changes in terms of reversal of irregularity and indentation. The second group with dominant stricture and persisting symptoms were considered for biliary drainage in the second phase of the study.

All except two of these patients under went biliary drainage procedures. Introperatively Collaterals in Hepatoduodenal ligament, level of stricture and bile duct stones were documented. Total blood loss and need for blood transfusion were recorded. Total three patients had marked collaterals in the operative area; however with careful ligation bleeding was avoided. One patient had BBS1, 4 had BBS2 and one had BBS type 3. Cholecystectomy was performed and Choleduchotomy was done close to hilum and stones were cleared from biliary tree. A satisfactory side to side Roux-en-Y hepatico jejunostomy was performed in all 6 patients. Average blood transfusion was 0.6 units. There was no post-operative mortality. Morbidity was mainly related to wound infection in two patients. There were no anastamotic leaks. Average hospital stay was 7.2 days. Progressive improvement was noted in LFT parameters in postoperative period.

All patients were followed up at 4 week interval for three months and every 3 months thereafter, with regular LFT and if needed ultra sound examination. One patient developed progressive liver failure and died after 4 months of second surgery. Other patients are being followed up till date and none required hospitalization for any biliary symptoms.

\section{Discussion}

Morphological changes in the bile ducts have been described in $80-100 \%$ of patients with EHPVO. However, clinical evidence of biliary obstruction (pain, jaundice, cholangitis) is seen in only $5-14 \%$ of patients (1-5). Biliary obstruction in patients with EHPVO is primarily due to compression of the bile duct by the enlarged paracholedochal plexus of veins $(1,2$, and 5). Ischemia secondary to thrombosis of small venules in the wall of the bile ducts has also been proposed as a cause of strictures. Patients with associated choledocholithiasis and recurrent episodes of cholangitis may also develop inflammatory strictures. ${ }^{[6,7]}$ The morphological pattern of biliary changes has been well documented by various authors (1-4, 6-7). Usually there is a long latent period for the development of biliary strictures after the onset of EHPVO. ${ }^{[8]}$ In our study the median duration of development of symptomatic biliary obstruction was 7.4 
Table 4: Summary of History and Investigations

\begin{tabular}{ll}
\hline Age & $\mathbf{2 2 - 4 5 y r s ~ ( m e a n ~ 3 8 . 4 y r s ) ~}$ \\
\hline Sex M:F & $5: 3$ \\
\hline Duration of symptoms & $6.75 \mathrm{yrs}$ \\
\hline History of intervention (stenting) & 4 patients \\
\hline Jaundice & Present in all during admission \\
\hline Cholangitis & Average-5 episodes \\
\hline CBD stone & All patients \\
\hline MRCP findings & BBS type1-1,type II-5,type III-1,type IV-1 \\
\hline Post operative liver biopsy & Advanced fibrosis in 2 patients \\
\hline
\end{tabular}

years after the diagnosis of EHPVO. Portal biliopathy can be managed by decompressing the collaterals in the hepatoduodenal ligament around the bile duct. Performing a PSRS achieves this and thus relieves the obstruction. ${ }^{[9-11]}$ Unlike other studies in our study, in patients without dominant biliary strictures, after portosystemic shunt showed successful resolution of the other abnormalities on follow-up. Chaudhary et al. have also described the usefulness of a PSRS in the management of strictures due to portal biliopathy. In their series, strictures resolved in three of five patients in whom PSRS was performed. However, the remaining two patients had persistent strictures at 6 months of follow up and required hepaticojejunostomy. In our series however none of the patients with dominant strictures responded to PSRS. Strictures that do not resolve following PSRS may be due to persistence of collaterals or perhaps secondary to ischemia. These patients require subsequent bilioenteric bypass or endoscopic dilation and stenting. Further need for biliary decompression following PSRS in such patients should be determined by the clinical course on follow up. The time interval required for resolution of biliary strictures following PSRS is not well documented. In series by Chaudhary et al 6 months was the time period. In a study by Ritu Khare et $\mathrm{al}^{\left[{ }^{[12]}\right.}$ repeat MRC at $12-15$ months showed resolution of strictures. In our study the MRC repeated after 6 weeks showed resolution in minor abnormalities, however this did not occur in dominant strictures. Two patients in our study because of delay in return to second surgery underwent MRC examination after 15 months without any change in the biliary abnormality, infact one of our patients had biliary stricture progressed from type 2 to type 3 after four month follow up. For decompression of the portal venous system, an alternative to PSRS is a transjugular intrahepatic portosystemic shunt (TIPPS). ${ }^{[13]}$ TIPPS in association with percutaneous clot dissolution and/or thrombectomy has usually been performed in the setting of acute portal vein thrombosis. There are a only a few reports of TIPPS in the setting of long-standing EHPVO with cavernomatous transformation.
The incidence of choledocholithiasis in patients with portal biliopathy has been reported to be $17 \% .{ }^{[7]}$ The higher (57 $\%$,) incidence in our study is probably because of inclusion of only symptomatic patients. CBD stones have been reported in EHPVO in the absence of gallstones. Biliary stasis and alterations in the composition of bile have been postulated as predisposing factors. In our study 5 out of the eight patients with choledocholithiasis had gallbladder stones. All these eight patients hailed from north India where there is a high prevalence of gallstone disease. It is possible that some of these patients had coexistent gallstone disease and EHPVO. Endoscopy has replaced surgery for the treatment of choledocholithiasis. Endoscopic extraction of stones is usually successful in patients with choledocholithiasis not associated with biliary stricture. Successful management of choledocholithiasis with endoscopic sphincterotomy and stone extraction has been previously described in EHPVO. ${ }^{[7,13]}$ Aggressive dilation and stone removal may cause hemobilia secondary to rupture of intramural varices. In our series there were no patients with choledocholithiasis alone. All these patients had underlying strictures. In our series, the previous attempt of ERC and stenting had failed in all these patients. Surgical intervention is a considerable undertaking in such patients considering the difficulty in accessing the bile ducts due to extensive pericholedochal collaterals. Massive intraoperative bleeding may be encountered in an attempt to explore the bile duct. Primary surgery of the biliary tract has been successful in only minority of patients. Severe hemorrhage during primary surgery on the biliary tract has been reported in other series. ${ }^{[11]}$ Therefore, the management strategy for patients with CBD stones and biliary strictures should be PSRS followed by bilioenteric bypass. In such situations, a staged procedure (PSRS followed by corrective biliary intervention subsequently) should be planned. The need for a PSRS after successful endoscopic extraction has been suggested to prevent stasis and recurrence of stones. ${ }^{[4]}$ The timing of biliary surgery following a PSRS is a matter of debate and no clear guidelines are available. ${ }^{[11,12]}$ In our series, 6 patients underwent a staged surgery at an interval 
of 2 and 15 months following PSRS. Severe bleeding was never encountered in any of these patients. Chaudhary et al. have performed biliary surgery after a variable interval of 9-12 months following PSS without any complications. A longer interval (up to 1 year) and documentation of a patent functioning shunt and decompression of collaterals by non-invasive imaging (color Doppler and MR angiography) may help in determining the optimum timing of surgery. The morbidity following biliary drainage was comparable to those of post cholecystectomy bile duct injury. This suggests that in all patients with dominant strictures needing surgical intervention our protocol should be followed. This is mainly because preoperative investigations are inadequate in delineating the magnitude of pericholedochal collaterals so a safe primary biliary drainage can be offered. Another interesting feature noted in our study was those patients with long duration of symptoms tend to have stone and stricture formation and are more prone for further complications. Hence early recognition of portal biliopathy and treatment by shunt surgery may prevent progression of biliary changes. However further studies are required to confirm this fact. The two mortalities in this series occurred in older patients with longer duration of symptoms and with biliary strictures. The cause of death was due to liver failure secondary to biliary cirrhosis. This also confirms that long duration of symptoms and severity of the disease are detrimental to prognosis and early intervention may save these patients. As endoscopic therapy is only symptomatic treatment and does not correct the underlying portal hypertension porta systemic decompression should be part of treatment of EHPVO with either symptomatic or asymptomatic portal biliopathy. Even though this statement cannot be generalized the above mentioned factor may be considered in treating patients with EHPVO for bleeding.

\section{Conclusion}

The patients with symptomatic portal biliopathy should be treated with porta systemic decompression and biliary drainage if necessary. Prevention of development of portal biliopathy may prompt surgery over endoscopic therapy for variceal bleeding in EHPVO. PSRS alone may be sufficient in minor biliary abnormalities in portal biliopathy. Persisting dominant strictures invariably need biliary drainage. This should be performed as early as 6 weeks to avoid complications of long standing biliary obstruction. Bilio enteric bypass is safe after PSRS with minimum morbidity. It is a single time treatment if performed properly and avoids repeat hospitalization and interventions of ERCP.

\section{References}

1. Sarin SK, Bhatia V, Makwane U. Portal biliopathy in extrahepatic portal vein obstruction. Ind J Gastroenterol.
1992;2:82-85.

2. Dilawari JB, Chawla YK. Pseudosclerosing cholangitis in extrahepatic portal venous obstruction. Gut. 1992;33(2):272276. Available from: https://dx.doi.org/10.1136/gut.33.2.272.

3. Bayraktar Y, Balkanci F, Kayhan B. Bile duct varices or 'pseudo-cholangiocarcinoma sign' in portal hypertension due to cavernous transformation of the portal vein. Am J Gastroenterol. 1992;87:1801-1806.

4. Condat B, Vilgrain V, Asselah T, O'Toole D, Rufat P, Zappa M. Portal cavernoma-associated cholangiopathy: A clinical and MR cholangiography coupled with MR portography imaging study. Hepatology. 2003;37(6):1302-1308. Available from: https://dx.doi.org/10.1053/jhep.2003.50232.

5. Khuroo MS, Yattoo GN, Zargar SA, Javid G, Dar MY, Khan BA, et al. Biliary abnormalities associated with extrahepatic portal venous obstruction. Hepatology. 1993;17(5):807-813. Available from: https://dx.doi.org/10.1002/hep.1840170510.

6. Malkan GH, Bhatia SJ, Bashir K, Khemani R, Abraham P, Gandhi MS. Cholangiopathy associated with portal hypertension: diagnostic evaluation and clinical implications. Gastrointest Endosc. 1999;49(3):344-348. Available from: https://dx.doi.org/10.1016/s0016-5107(99)70011-8.

7. Bhatia V, Jain AK, Sarin SK. Choledocholithiasis associated with portal biliopathy in patients with extrahepatic portal vein obstruction: Management with endoscopic sphincterotomy. Gastrointest Endosc. 1995;42(2):178-181. Available from: https://dx.doi.org/10.1016/s0016-5107(95)70080-3.

8. Chandra R, Tharakan A, Kapoor D, Sarin SK. Comparative study of portal biliopathy in patients with portal hypertension due to different etiologies. Ind J Gastroenterol. 1997;15:59-62.

9. Choudhuri G, Tandon RK, Nundy S, Misra NK. Common bile duct obstruction by portal cavernoma. Dig Dis Sci. 1988;33(12):1626-1628. Available from: https://dx.doi.org/10. 1007/bf01535956.

10. Dhiman RK, Puri P, Chawla Y, Minz M, Bapuraj JR, Gupta S, et al. Biliary changes in extrahepatic portal venous obstruction: compression by collaterals or ischemic? Gastrointest Endosc. 1999;50(5):646-652. Available from: https://dx.doi.org/10. 1016/s0016-5107(99)80013-3.

11. Chaudhary A, Dhar P, Sarin SK, Sachdev A, Agarwal AK, Vij JC. Bile duct obstruction due to portal biliopathy in extrahepatic portal hypertension: surgical management. $\mathrm{Br} \mathrm{J}$ Surg. 1998;85(3):326-329. Available from: https://dx.doi.org/ 10.1046/j.1365-2168.1998.00591.x.

12. Khare R, Sikora SS, Srikanth G, Choudhuri G, Saraswat VA, Kumar A, et al. Extrahepatic portal venous obstruction and obstructive jaundice: Approach to management. J Gastroenterol Hepatol. 2005;20(1):56-61. Available from: https://dx.doi.org/ 10.1111/j.1440-1746.2004.03528.x.

13. Kawamata H, Kumazaki T, Kanazawa H, Takahashi S, Tajima $\mathrm{H}$, Hayashi H. Transjugular intrahepatic portosystemic shunt in a patient with cavernomatous portal vein occlusion. Cardiovasc Intervent Radiol. 2000;23(2):145-149. Available from: https: //dx.doi.org/10.1007/s002709910030. 
Copyright: (C) the author(s), 2020. It is an open-access article distributed under the terms of the Creative Commons Attribution License (CC BY 4.0), which permits authors to retain ownership of the copyright for their content, and allow anyone to download, reuse, reprint, modify, distribute and/or copy the content as long as the original authors and source are cited.

How to cite this article: Girish SP, Reddy JMB. Surgical Management of Portal Biliopathy - A Prospective Analysis.. Acad. J Surg. 2020;3(1):176-182.

DOI: dx.doi.org/10.47008/ajs/2020.3.1.38

Source of Support: Nil, Conflict of Interest: None declared. 